\title{
DETERMINAÇÃO CONDUTOMÉTRICA DE CLORIDRATO DE METFORMINA EM FORMULAÇÕES FARMACÊUTICAS EMPREGANDO NITRATO DE PRATA COMO TITULANTE
}

\author{
Elen Romão Sartori, Willian Toito Suarez e Orlando Fatibello-Filho* \\ Departamento de Química, Universidade Federal de São Carlos, CP 676, 13560-970 São Carlos - SP, Brasil
}

Recebido em 26/9/08; aceito em 5/3/09; publicado na web em 4/8/09

\begin{abstract}
CONDUCTOMETRIC DETERMINATION OF METFORMIN HYDROCHLORIDE IN PHARMACEUTICAL FORMULATIONS USING SILVER NITRATE AS TITRANT. A simple, precise, rapid and low-cost conductometric titration method for the determination of metformin hydrochloride (MET) in pharmaceuticals using silver nitrate as titrant is proposed. The method was based on the chemical reaction between the chloride of metformin hydrochloride molecule and $\mathrm{Ag}(\mathrm{I})$ ions, yielding the precipitate $\mathrm{AgCl}_{(\mathrm{s})}$. The method was applied for MET determination in three pharmaceuticals and the obtained results with proposed method were in close agreement with those results obtained using an official method of the British Pharmacopoeia, at a 95\% confidence level.
\end{abstract}

Keywords: conductometry titration; metformin hydrochloride; silver nitrate.

\section{INTRODUÇÃO}

O diabetes mellitus (DM) é uma doença crônica, caracterizada pela elevação da glicose no sangue acima da taxa normal (hiperglicemia), que é de aproximadamente 70 a $100 \mathrm{mg} \mathrm{dL} \cdot{ }^{1,2} \mathrm{O}$ diabetes mellitus tipo 2 é provocado predominantemente por um estado de resistência à ação da insulina associada a uma relativa deficiência de sua secreção pelo metabolismo. Pessoas inicialmente classificadas com este tipo de diabetes, principalmente obesas, hipertensas e sedentárias, não precisam de insulina injetável para manter um nível ideal de glicose no sangue, contrário ao diabetes mellitus tipo 1, cuja aplicação de insulina é a única forma de tratamento. ${ }^{3}$

A prevenção ou retardamento do diabetes mellitus tipo 2 implica na prática de uma atividade física regular e uma alimentação com redução de açúcares e calorias em associação ao uso diário de um medicamento antidiabético oral, o que torna usual a prescrição do cloridrato de metformina (MET), que constitui um dos medicamentos de escolha inicial tradicional para este tipo de diabetes. ${ }^{3,4}$

O cloridrato de metformina é uma droga com propriedades anti-hiperglicêmicas que normaliza os níveis elevados de glicose no sangue. Ele aumenta a sensibilidade à insulina no músculo esquelético, tecido adiposo e, especialmente, no fígado, reduzindo a gliconeogênese hepática e aumentando a captação periférica de glicose. ${ }^{4}$ Sua fórmula estrutural, 1,1-dimetilbiguanida, é mostrada na Figura $1 .^{3}$<smiles>CN(C)C(=N)NC(=N)N</smiles>

Figura 1. Fórmula estrutural do cloridrato de metformina

Devido à importância farmacológica do cloridrato de metformina no tratamento do diabetes tipo 2, há um interesse no desenvolvimento de métodos analíticos simples e viáveis para a determinação deste analito em produtos farmacêuticos.

Diversos métodos analíticos são descritos na literatura para a

*e-mail: bello@ufscar.br determinação quantitativa de MET em formulações farmacêuticas. Estes procedimentos incluem: espectrofotometria, ${ }^{5-7}$ cromatografia líquida de alta eficiência (CLAE), ${ }^{8}$ potenciometria, ${ }^{6,9-11}$ voltametria, ${ }^{12-14}$ entre outros. ${ }^{6,15,16}$ Muitos desses métodos não são simples para análises de rotina. Outros métodos são morosos e empregam reagentes orgânicos, além de instrumentos de alto custo e/ou sofisticados.

Martínez-Calatalyud et al. ${ }^{17}$ determinaram condutometricamente cloridrato de metformina em preparações farmacêuticas empregando sulfato de cobre(II) como titulante. Nesse procedimento, a formação do complexo cor-de-rosa de $\mathrm{CuMET}_{2}{ }^{2+}$ é observada em meio aquoso contendo a mistura de $\mathrm{NaOH} 0,06 \mathrm{~mol} \mathrm{~L}^{-1} / \mathrm{NH}_{3}$ $0,1 \mathrm{~mol} \mathrm{~L}^{-1} 1: 15 \mathrm{v} / \mathrm{v}(\mathrm{pH} 11,0-11,5)$ a $20^{\circ} \mathrm{C}$, sendo que pequenas variações do meio reacional podem diminuir a exatidão do método. Em um outro método condutométrico, ${ }^{18} \mathrm{MET}$ foi titulado com uma solução de tetrafenilborato de sódio e brometo de cetilpiridina, sendo que o emprego desse titulante não é recomendado, devido sua alta toxicidade.

No presente trabalho descreve-se a titulação condutométrica do cloridrato de metformina empregando-se nitrato de prata como titulante. A reação baseia-se na formação de um sal insolúvel $\left(\mathrm{AgCl}_{(\mathrm{s})}\right)$ entre o titulante $\mathrm{AgNO}_{3(\mathrm{aq})}$ e o cloreto do cloridrato $(\mathrm{HCl})$ da molécula de metformina. O método foi aplicado na determinação de MET em amostras comerciais e comparado ao método espectrofotométrico descrito pela Farmacopéia Britânica. ${ }^{5}$

\section{PARTE EXPERIMENTAL}

\section{Material e equipamentos}

Para as medidas condutométricas utilizou-se uma microbureta automática Methrom/Herisau, modelo E274, um condutivímetro Micronal, modelo B330, célula de vidro com camisa dupla para o controle da temperatura e célula condutométrica Digimed DMC-010.

Para o método comparativo utilizou-se um espectrofotômetro com arranjo linear de diodos Hewlett Packard, modelo 8452A, empregando-se uma cubeta de quartzo de 1,0 cm de caminho óptico acoplada a um microcomputador.

O programa OriginPro 6.0 foi usado para a confecção dos gráfi$\cos$. Todas as medidas foram feitas com agitação constante da solução e a uma temperatura controlada de $25 \pm 1{ }^{\circ} \mathrm{C}$. 


\section{Reagentes e soluções}

Todos os reagentes empregados foram de grau analítico e todas as soluções foram preparadas com água desionizada obtida de um sistema Milli-Q Millipore (Bedford, MA), modelo UV Plus ultrabaixo teor de substâncias orgânicas dissolvidas. Foram utilizados cloridrato de metformina padrão (Sigma) e $\mathrm{AgNO}_{3}$ (Aldrich).

A solução estoque de $\mathrm{AgNO}_{3} 2,0 \times 10^{-2} \mathrm{~mol} \mathrm{~L}^{-1}$ foi preparada dissolvendo-se uma massa adequada deste sal em água desionizada. As soluções de nitrato de prata variando de $5,0 \times 10^{-4}$ a $1,0 \times 10^{-2} \mathrm{~mol} \mathrm{~L}^{-1}$ foram obtidas diluindo-se apropriadamente a solução estoque.

As soluções de cloridrato de metformina no intervalo de 5,0 $\times 10^{-5}$ a $1,0 \times 10^{-2} \mathrm{~mol} \mathrm{~L}^{-1}$ foram preparadas diluindo-se uma solução estoque de concentração $2,0 \times 10^{-2} \mathrm{~mol} \mathrm{~L}^{-1}$.

\section{Determinação de cloridrato de metformina em amostras comerciais}

Amostras, na forma de comprimido, contendo cloridrato de metformina foram adquiridas no comércio local. Dez comprimidos de cada amostra foram triturados e para o preparo da solução foi utilizada uma massa equivalente a um comprimido, que foi dissolvida em 100 $\mathrm{mL}$ de água desionizada. Os sólidos não dissolvidos foram filtrados em papel de filtro Whatman № 1 e, então, alíquotas de $10 \mathrm{~mL}$ da solução filtrada foram tituladas com solução de $\mathrm{AgNO}_{3}$.

\section{Procedimento comparativo}

O procedimento espectrofotométrico descrito na Farmacopéia Britânica ${ }^{5}$ foi aplicado como método de comparação ao procedimento proposto. Cinco comprimidos de cada amostra foram pesados e pulverizados em almofariz. Uma massa equivalente a $0,1 \mathrm{~g}$ de cloridrato de metformina foi dissolvida em balão volumétrico de $100 \mathrm{~mL}$, completando-se o volume com água desionizada. Posteriormente essa solução foi filtrada e uma alíquota de $10 \mathrm{~mL}$ do filtrado foi diluída para $100 \mathrm{~mL}$ com água desionizada. Por fim, uma alíquota de $10 \mathrm{~mL}$ dessa solução resultante foi diluída para $100 \mathrm{~mL}$ também com água desionizada e mediu-se a absorbância em $232 \mathrm{~nm}$, utilizando água para ajuste do branco. Calculou-se o teor de MET nos comprimidos adotando-se $798 \mathrm{~cm}^{-1} \mathrm{~mol}^{-1} \mathrm{~L}$ como o valor da absortividade molar.

\section{Titulação condutométrica}

Alíquotas de $10 \mathrm{~mL}$ (soluções de referência ou da amostra) foram transferidas para uma célula de vidro com camisa dupla e tituladas com soluções de mesma concentração de nitrato de prata, registrando-se a cada adição do titulante, em intervalos de 15 s, o valor da condutância.

$\mathrm{O}$ valor da condutância obtida deve ser corrigido em função do volume de titulante adicionado antes de se traçar o gráfico, ${ }^{19}$ de acordo com a Equação 1, sendo: $\mathrm{L}_{\text {corr }}$ a condutância corrigida, $\mathrm{L}_{\exp }$ a condutância experimental, $\mathrm{V}_{\mathrm{i}}$ o volume inicial e $\mathrm{V}_{\mathrm{a}}$ o volume adicionado.

$\mathrm{L}_{\text {corr }}=\mathrm{L}_{\text {exp }} \times\left(\left(\mathrm{V}_{\mathrm{i}}+\mathrm{V}_{\mathrm{a}}\right) / \mathrm{V}_{\mathrm{i}}\right)$

A partir do gráfico de condutância vs o volume de solução de $\mathrm{AgNO}_{3}$, encontrou-se o volume equivalente e, assim, a concentração de MET nas soluções tituladas.

\section{RESULTADOS E DISCUSSÃO}

\section{Estudos preliminares}

Inicialmente, avaliou-se o intervalo de tempo de 10, 15, 20 e $30 \mathrm{~s}$ entre as adições do titulante utilizando-se concentrações de MET $1,03 \times 10^{-2} \mathrm{~mol} \mathrm{~L}^{-1}$ e de $\mathrm{AgNO}_{3} 1,05 \times 10^{-2} \mathrm{~mol} \mathrm{~L}^{-1}$, a fim de se obter maior precisão nos resultados. $\mathrm{O}$ melhor resultado foi obtido com o intervalo de tempo de $15 \mathrm{~s}$ entre as medidas, pois este apresentou um menor desvio padrão relativo entre as curvas de titulação e, consequentemente, uma melhor resolução.

Com o tempo estabelecido em $15 \mathrm{~s}$, realizaram-se três titulações sucessivas de solução padrão de MET em concentrações variando de $5,02 \times 10^{-4}$ a $1,02 \times 10^{-2} \mathrm{~mol} \mathrm{~L}^{-1}$ utilizando o titulante nitrato de prata sempre na mesma concentração do titulado.

A Tabela 1 apresenta os resultados obtidos para cada concentração de solução padrão (de referência) de cloridrato de metformina. Há uma boa concordância entre os teores de MET determinados condutometricamente e as concentrações das soluções de referência, a um nível de confiança de $95 \%$.

Tabela 1. Comparação entre os teores conhecidos de cloridrato de metformina na solução de referência e aqueles determinados empregando-se o método condutométrico proposto, a $25^{\circ} \mathrm{C}$

\begin{tabular}{lcc}
\hline $\begin{array}{l}\text { Soluções de referência de } \\
\text { MET }\left(\mathrm{mol} \mathrm{L}^{-1}\right)\end{array}$ & Experimental $\left(\mathrm{mol} \mathrm{L}^{-1}\right)^{*}$ & Erro $(\%)$ \\
\hline $5,0 \times 10^{-4}$ & $(5,0 \pm 0,3) \times 10^{-4}$ & 0 \\
$1,0 \times 10^{-3}$ & $(1,0 \pm 0,1) \times 10^{-3}$ & 0 \\
$5,0 \times 10^{-3}$ & $(5,1 \pm 0,2) \times 10^{-3}$ & 2,0 \\
$1,0 \times 10^{-2}$ & $(1,0 \pm 0,1) \times 10^{-2}$ & 0 \\
\hline
\end{tabular}

* $\mathrm{n}=3$; média \pm desvio padrão.

Para concentrações de MET menores de 5,02 × 10-4 $\mathrm{mol} \mathrm{L}^{-1}$ a adição do titulante ocasionou pequenas variações de condutância, tornando não confiável a determinação do ponto final da titulação. Logo, a determinação do ponto de equivalência pode ser determinada até um teor mínimo de 5,02 × $10^{-4} \mathrm{~mol} \mathrm{~L}^{-1}$, com uma exatidão menor, da ordem de 6-7\%. Isto ocorre devido à diluição das soluções e à solubilidade do $\mathrm{AgCl}_{(\mathrm{s})}$ formado.

A Figura 2 apresenta uma curva condutométrica característica obtida para a determinação de $10 \mathrm{~mL}$ de cloridrato de metformina $1,02 \times 10^{-2} \mathrm{~mol} \mathrm{~L}^{-1}$ utilizando solução de $\mathrm{Ag}^{+} 1,0 \times 10^{-2} \mathrm{~mol} \mathrm{~L}^{-1}$ como titulante.

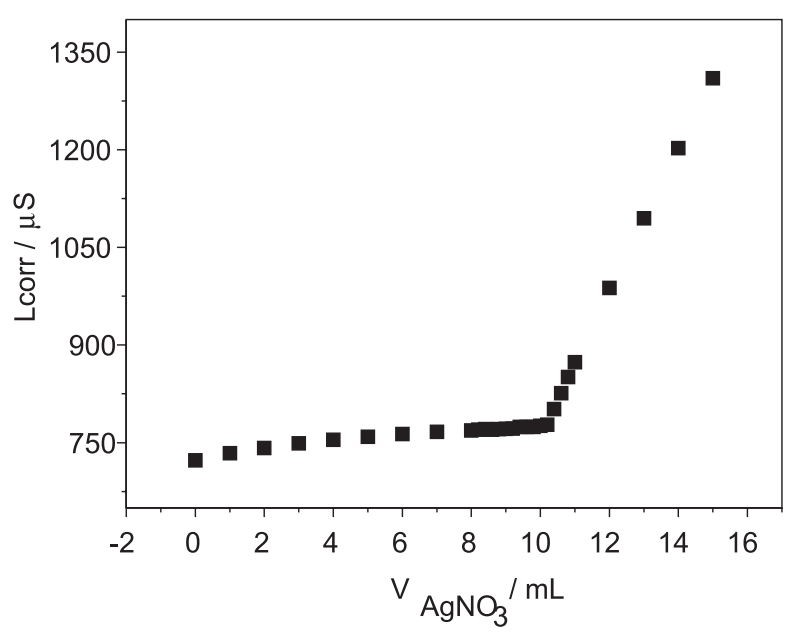

Figura 2. Curva da titulação condutométrica de cloridrato de metformina 1,02 $\times 10^{-2} \mathrm{~mol} \mathrm{~L}^{-1}$ utilizando solução de $\mathrm{AgNO}_{3} 1,00 \times 10^{-2} \mathrm{~mol} \mathrm{~L}^{-1}$ como titulante 
A condutância medida antes da adição do titulante (volume de $\mathrm{AgNO}_{3}$ igual a zero) deve-se à molécula de metformina presente na cela condutométrica.

Até o ponto de equivalência, a titulação envolve a precipitação dos íons $\mathrm{Cl}^{-}$com $\mathrm{Ag}^{+}$, formando o precipitado de $\mathrm{AgCl}_{(\mathrm{s})}$ (Figura 3), que possui baixa solubilidade $\left(1,1 \times 10^{-5} \mathrm{~mol} \mathrm{~L}^{-1}\right) .{ }^{19}$ Devido à alta condutividade iônica dos íons $\mathrm{H}^{+}\left(\mathrm{H}_{3} \mathrm{O}^{+}\right)$era de se esperar que o primeiro ramo da curva condutométrica possuísse uma inclinação maior do que a observada experimentalmente (Figura 3). Provavelmente, à medida que os íons cloreto vão sendo titulados, os íons $\mathrm{H}^{+}\left(\mathrm{H}_{3} \mathrm{O}^{+}\right)$ podem estar protonando os grupos aminos da molécula de metformina $\mathrm{e}$, consequentemente, diminuindo a inclinação do primeiro ramo da curva de titulação, atribuída à pequena concentração de íons $\mathrm{H}_{3} \mathrm{O}^{+}{ }_{(\mathrm{aq})}$ livres na solução e também ao aumento da concentração dos íons nitrato do titulante.

Após o ponto de equivalência, à medida que são adicionados volumes crescentes da solução do titulante, há um aumento pronunciado da condutância em função do excesso de volume da solução de $\mathrm{AgNO}_{3(\mathrm{aq})}$ adicionado. $\mathrm{O}$ aumento das concentrações desse cátion metálico e dos ânions $\mathrm{NO}_{3 \text { (aq) }}^{-}$na solução resultante promove o aumento da inclinação do segundo ramo da curva de titulação. O ponto final da titulação foi determinado pelo ponto de intersecção dos dois segmentos da reta.

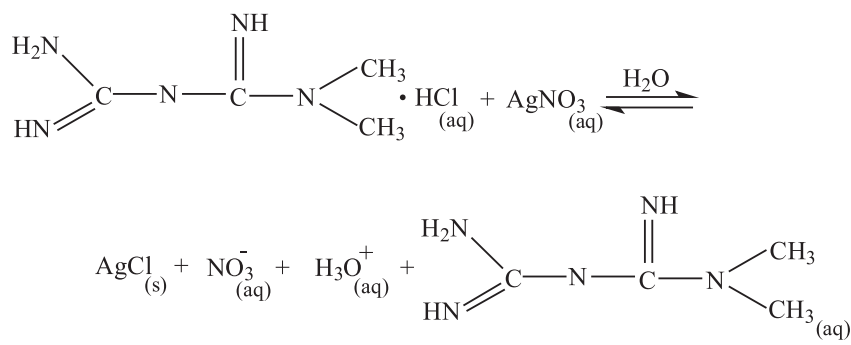

Figura 3. Reação envolvida na titulação condutométrica de cloridrato de metformina com $\mathrm{AgNO}_{3}$

No estudo da repetibilidade do procedimento condutométrico, uma solução de MET $1,10 \times 10^{-2} \mathrm{~mol} \mathrm{~L}^{-1}$ foi titulada condutometricamente por seis vezes $(n=6)$ com solução de $\mathrm{AgNO}_{3} 1,09 \times 10^{-2} \mathrm{~mol}$ $\mathrm{L}^{-1}$ e o desvio padrão relativo (RSD) obtido foi menor que $0,8 \%$.

\section{Estudo de interferentes em potencial}

O efeito de interferentes em potencial na determinação de MET em formulações farmacêuticas foi avaliado para excipientes normalmente presentes em amostras comerciais (povidona, estearato de magnésio, celulose, polietilenoglicol, dióxido de titânio, parafina e sorbitol). Nesses experimentos foram feitas comparações do volume do ponto de equivalência obtido empregando-se a mesma solução de referência de MET 1,05 × 10-2 $\mathrm{mol} \mathrm{L}^{-1}$ com o volume do ponto de equivalência obtido, empregando-se a mesma solução de referência de MET juntamente com cada um dos interferentes em potencial nas concentrações esperadas nas formulações farmacêuticas. Não houve interferência significativa nos valores de volume de equivalência encontrados para esses interferentes estudados. A maioria destes excipientes é insolúvel e/ou possui baixa solubilidade.

\section{Teste de recuperação}

O estudo de adição e recuperação do analito foi feito com três diferentes concentrações de cloridrato de metformina, adicionandose solução padrão (referência) de MET a três diferentes amostras de formulações farmacêuticas de diferentes dosagens, 500, 850 e 1000 $\mathrm{mg}$ /comprimido. Os resultados foram comparados com aqueles obtidos com as amostras não adicionadas de padrão. As recuperações de MET variaram de 99,0 a 105\%, indicando que não há interferência significativa da matriz nas amostras analisadas.

\section{Aplicações}

O método proposto foi aplicado na determinação de cloridrato de metformina em três diferentes formulações farmacêuticas de diferentes dosagens e os resultados foram comparados com aqueles obtidos empregando-se o método espectrofotométrico recomendado pela Farmacopéia Britânica ${ }^{5}$ e pelos valores rotulados. A Tabela 2 apresenta os resultados obtidos para essas determinações.

Tabela 2. Teores de cloridrato de metformina obtidos empregandose o método espectrofotométrico recomendado pela Farmacopéia Britânica $^{5}$ e a titulação condutométrica

\begin{tabular}{lccccc}
\hline & \multicolumn{3}{c}{ MET (mg/comprimido) } & \multicolumn{2}{c}{ Erro relativo (\%) } \\
Amostras & Rotulado $\begin{array}{c}\text { Método de } \\
\text { referência }{ }^{a}\end{array}$ & $\begin{array}{c}\text { Titulação } \\
\text { conduto- } \\
\text { métrica }\end{array}$ & $\operatorname{Re}_{1}{ }^{b}$ & $\operatorname{Re}_{2}{ }^{c}$ \\
\hline $\mathrm{A}$ & 500 & $497 \pm 7$ & $501 \pm 5$ & 0,2 & 0,8 \\
$\mathrm{~B}$ & 850 & $851 \pm 5$ & $849 \pm 6$ & $-0,1$ & $-0,2$ \\
$\mathrm{C}$ & 1000 & $1020 \pm 12$ & $1021 \pm 14$ & 2,1 & 0,1 \\
\hline${ }^{a} \mathrm{n}=3 ;{ }^{b} \operatorname{Re}_{1}=100 \times($ titulação condutométrica - valor rotulado) / \\
valor rotulado; ${ }^{c} \operatorname{Re}_{2}=100 \times($ titulação condutométrica - método de \\
referência) / método de referência.
\end{tabular}

O teste-t pareado ${ }^{20}$ foi aplicado aos resultados obtidos empregando ambos os métodos, resultando em um valor de t calculado $(2,730)$ menor que o valor de $t$ crítico $(4,303)$, indicando que o procedimento proposto possui boa exatidão, uma vez que não foi observada diferença significativa entre os resultados obtidos, a um nível de confiança de $95 \%$.

\section{CONCLUSÕES}

O método condutométrico proposto para a determinação de cloridrato de metformina em formulações farmacêuticas é simples, preciso, exato e de baixo custo. Ademais, as amostras foram analisadas sem a necessidade de tratamento especial ou do emprego de algum tipo de reagente orgânico, muitas vezes de média ou alta toxicidade, podendo assim ser aplicado em laboratórios de análise de rotina.

\section{AGRADECIMENTOS}

À CAPES (Coordenação de Aperfeiçoamento de Pessoal de Nível Superior), ao CNPq (Conselho Nacional de Desenvolvimento Científico e Tecnológico) e à FAPESP (Fundação de Amparo à Pesquisa do Estado de São Paulo) pelo suporte financeiro e pelas bolsas concedidas.

\section{REFERÊNCIAS}

1. http://www.diabetes.org.br, acessada em Setembro 2008.

2. Ramalho, A. C. R. Em Farmacologia; Silva, P., ed.; 6 ed., Guanabara Koogan: Rio de Janeiro, 2002, cap. 82.

3. Nolte, M. S.; Karan, J. H. Em Farmacologia Básica e Clínica; Katzung, B. G., ed.; 9a ed., Guanabara Koogan: Rio de Janeiro, 2006, cap. 41. 
4. Branchtein, L.; Matos, M. C. G. Em Farmacologia Clínica: Fundamentos da Terapêutica Reacional; Fuchs, F. D.; Wannmacher, L.; Ferreira, M. B., eds.; $3^{\text {a }}$ ed., Guanabara Koogan: Rio de Janeiro, 2004, cap. 63.

5. British Pharmacopoeia, HM Stationery Office: London, 1998, p. 1802.

6. Hassan, S. S. M.; Mahmoud, W. H.; Elmosallamy, M. A. F.; Othaman, A. H.; Anal. Chim. Acta 1999, 378, 299.

7. Ashour, S.; Kabbani, R.; Anal. Lett. 2003, 36, 361.

8. Vasudevan, M.; Ravi, J.; Ravisankar, S.; Suresh, B.; J. Pharm. Biom. Anal. 2001, 25, 77 .

9. European Pharmacopoeia, Council of Europe: Strasbourg, 2000, p. 937.

10. Rizk, M. S.; Abbel-Fattah, H. M.; Issa, Y. M.; Atia, E. M.; Anal. Lett. 1993, 26, 415 .
11. Khaled, E.; Hassan, H. N. A; Kamel, M. S; Barsoum, B. N.; Current Pharm. Anal. 2007, 3, 262.

12. Liu, Y. G.; Li, G. Z.; Chinese J. Anal. Chem. 2001, 29, 1027.

13. Tian, X.; Song, J.; Luan, X-j.; Wang, Y-y.; Shi, Q-z.; Anal. Bional. Chem. 2006, 386, 2081.

14. Tian, X.; Song, J.; J. Pharm. Biom. Anal. 2007, 44, 1192.

15. Yardimci, C.; Özaltin, N.; Anal. Chim. Acta 2005, 549, 88.

16. Habib, I. H. I.; Kamel, M. S.; Talanta 2003, 60, 185

17. Martinez Calatayud, J.; Campíns Falco, P.; Pascual Martí, M. C.; Anal. Lett. 1985, 18, 1385.

18. Abou-Dan, M.; Ashour, S.; Adou-Dan, H.; Asian J. Chem. 2001, 13, 1.

19. Jeffery, G. H.; Basset, J.; Mendham, J.; Denney, R. C.; Vogel's Quantitative Chemical Analysis, $5^{\text {th }}$ ed., Longman Scientific \& Technical: New York, 1989.

20. Anderson, R. L.; Practical Statistics for Analytical Chemists, D. Van Nostrand Reinhold Company: New York, 1987. 